gendlichen nicht in derselben Weise könnten. Die Mediennutzung als den zentralen Indikator für Individualisierung und Bewältigung der „Moderne“ zu definieren, wird zudem durch die Entwicklung der Medien selbst relativiert: Ihre Ubiquität und ihre fortschreitende Diversifizierung ist mit Prozessen der Normalisierung und Banalisierung verbunden. Medien haben auch eine banale, triviale Seite, sie sind Teil des Alltag der Jugendlichen. Im Übrigen: Medien als solche sind nicht immer und $\mathrm{zu}$ jedem historischen Zeitpunkt ein Medium der „Moderne“ - wie ein Blick z. B. auf die Rolle der Medien nach 1945 in West - und Ostdeutschland zeigt.

Diese Untersuchung leistet eine gründliche Auseinandersetzung mit den theoretischen und empirischen Desideraten der sozialwissenschaftlichen Jugendforschung. Die Ergebnisse der Medienforschung werden diskutiert. Das Vorhaben, eine vergleichende Untersuchung west- und ostdeutscher Jugendlicher unter Berücksichtigung soziokultureller Aspekte anzugehen, ist verdienstvoll, weil es offenbar an dergleichen mangelt. Die Ergebnisse belegen Gemeinsamkeiten aber auch Unterschiede in der Zuwendung und in der Bewertung von Medien zwischen Ost und West. Der Hinweis auf die erklärungsstarken soziokulturellen Bedingungen macht generell den Mangel an Untersuchungen deutlich, die sich mit den kulturellen Differenzen zwischen Regionen, nicht nur denen zwischen Ost und West, beschäftigen. Es ist das Verdienst dieser Untersuchung, dass ihre Fragestellungen und Ergebnisse zugleich ein Ausgangspunkt für eine regional-vergleichende Medienforschung sein könnte.

Ekkehard Sander

\section{Beck'scher TKG-Kommentar}

Hrsg. von Wolfgang Büchner et. al

München: Beck, 2. Aufl., 2000. - 1734 S.

ISBN 3-406-45427-5

Der Beck'sche Kommentar zum Telekommunikationsgesetz bewegt sich mittlerweile selbst wie die Unternehmen in dem von ihm betrachteten Markt in einem hoch kompetativen Umfeld. Es ist dennoch erwartbar, dass er eine starke Marktmacht behält, und das nicht nur, weil er mittlerweile als der etablierteste Kommentar im Telekommunikationsrecht gelten kann. Dazu trägt zum einen die klare, für alle Normen zumindest ähnliche Gliederung der Kommentierung bei. Zum anderen merkt man dem Autorenteam - überwiegend in diesem Bereich ausgewiesene Rechtsanwälte und Justitiare von Telekommunikationsunternehmen - ihre dauerhafte praktische Befassung mit der Materie an. Kaum ein relevantes Problemfeld, das nicht im Kommentar Erwähnung findet; keine Beschränkung auf die Auswertung von Literatur und gefestigter Rechtsprechung, sondern die Berücksichtigung von zahlreichen Entscheidungen der Regulierungsbehörde für Telekommunikation und Post stärkt die Argumentation in den einzelnen Kommentierungen und zeichnet den Kommentar aus.

Die zweite Auflage macht allerdings deutlich, dass ein solcher, von Einzelfällen in der Praxis her synthetisierender Ansatz bei einem dynamischen Feld wie dem Telekommunikationsrecht, das darüber hinaus auch noch durch europarechtliche Einflüsse an Komplexität gewinnt, seinen Preis hat. Es wird zunehmend schwieriger, sich in den zum Teil über eine Seite ziehenden Absätzen die relevanten Informationen zu erschließen. Grundsätze, die die Auslegung leiten und die Darstellung strukturieren können, werden nicht an allen Stellen sichtbar.

Dies ändert allerdings nichts an der Gesamtbewertung: Ohne den Beck'schen TKG-Kommentar kann man schlicht in diesem Rechtsbereich nicht seriös arbeiten. Vielleicht gibt die anstehende Novellierung des TKG Gelegenheit, die vielfältigen, in den Kommentar eingehenden Erfahrungen noch konsequenter zu bündeln und so für den Nutzer noch einfacher erschließbar zu machen.

Wolfgang Schulz

\section{Werner Früh}

\section{Gewaltpotentiale des Fernsehangebots}

Programmangebot und zielgruppenspezifische Interpretation

Wiesbaden: Westdeutscher Verlag, 2001. $221 \mathrm{~S}$.

ISBN 3-531-13706-9

Ausgangspunkt der Studie von Werner Früh ist die wissenschaftliche Debatte um die Wirkung von Gewaltdarstellungen im Fernsehen. Einer der grundlegenden Streitpunkte in dieser De- 